\title{
Patient-Specific Instrumentation Combined With A New Tool for Gap Balancing Is Useful in Total Knee Replacement: 3-Year Follow-Up of A Retrospective Study
}

\section{Ting Deng}

the thrid hospital of changsha https://orcid.org/0000-0002-8311-6820

\section{Tangyou Liu}

the Thrid Hospital of Changsha

Qing Lei ( $D$ lqing0504@hotmail.com )

the thrid hospital of changsha https://orcid.org/0000-0002-0344-8754

Lihong Cai

the thrid hospital of changsha

\section{Song Chen}

the thrid hospital of changsha

\section{Research article}

Keywords: Total knee arthroplasty, Gap balance, Ligament balance, Balancer device, Flexion-extension gap Surgical technique, Equipment design, Implantation technique, Measured resection technique

Posted Date: December 18th, 2020

DOI: https://doi.org/10.21203/rs.3.rs-129846/v1

License: (c) (i) This work is licensed under a Creative Commons Attribution 4.0 International License.

Read Full License 


\section{Abstract}

ObjectiveXThe purpose of this study was to prove that knee function is well recovered using gap balancing technique with patient-specific instrumentation $\triangle \mathrm{PSI} \llbracket$ combined a new balancer device in total knee arthroplasty (TKA) compared with the measured resection technique.

Materials and methods $\varangle$ Data of 152 patients received TKA from August 2014 to June 2016 were studied retrospectively. Gap balancing technique assisted by PSI combined a new balance device was used in 80 patients (82 knees) and the measured resection technique was used in the surgery in 70 patients (70 knees). The data of surgery, imaging and knee function were compared.

Results囚The gap balancing technique assisted by PSI and a new balancer device was feasible in all operated knees and found to be reliable. In total, 152 patients ( 150 knees) with ages ranging from 52 to 78 years (mean 67 years) underwent TKA during the study period. The follow-up ranged from 35 to 52 months (mean 45 months). Only one patient underwent revision surgery in gap balance group at 2 years postoperatively due to infection. There was no difference in the incidence of anterior knee pain between the two groups. 2 patients received or required revisions. Until the latest follow-up, the mean flexion

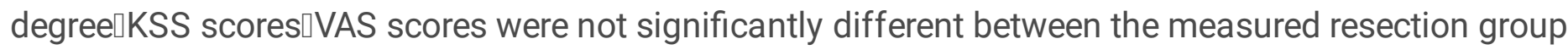
and gap-balancing group at 12 weeks and 36 weeks. The average joint line displacement in GB group was $1.3 \pm 1.1 \mathrm{~mm}$ (range $0-3$ ) proximal and $1.2 \pm 1.4 \mathrm{~mm}$ in MR group. No outliers $₫ 5 \mathrm{~mm}$ in each group were recorded. The mean leg axis was $1.8^{\circ} \pm 1.5^{\circ}$ varus (range $0^{\circ}-3^{\circ}$ varus) versus the neutral mechanical axis in GB group and $1.4^{\circ} \pm 1.2^{\circ}$ (range $0^{\circ}-3^{\circ}$ ) in MR group. No outliers with $\otimes 3^{\circ}$ deviation in each group were recorded.

Conclusions区The gap balancing technique assisted by the new balancer device and PSI can be used to achieve accurate femoral component alignment as well as measured resection in 3 years outcomes. The new balancer device can be taken into account by surgeons who prefer the gap-balancing technique together with the PSI.

\section{Introduction}

Total knee arthroplasty (TKA) is considered to be the most successful surgical method in the 21 st century. It is an effective procedure for end-stage knee osteoarthritis. A successful knee replacement hinges on appropriate soft-tissue balancing and accurate bony alignment. An ideal limb alignment and soft-tissue balancing may allow patients to gain the function close to the normal knee, avoiding the early failure of TKA caused by uneven force bearing of prosthesis and cement, and maximum limit assures the integrity of extensor mechanism. The discussions of TKA surgical technique focus on how to determine femoral component rotation. The femoral component rotational malalignment may lead to patellofemoral complications,such as abnormal patellar tracking $\square$ knee anterior pain $\square$ joint adhesion $\square$ and all these can increase instability, poor function and wear[1-3]. 
The rotational alignment of the femoral component references to bony anatomy and soft tissue[4, 5]. There are two standard surgical techniques for prosthesis implantation utilized in TKA: measured resection and gap balancing.

Measured resection technique maintains stable postoperative joint line with short learning curve, simple operation. Most surgeons used bony landmarks such as the Transepicondylar Axis, Anteroposterior Axis or Posterior Condylar Axis to set femoral component rotation when using a measured resection technique[6]. Some studies believe that measured resection technique is inaccurate due to variations in femoral anatomy-it's difficult to determine femoral component rotation by TEA, the AP axis or the posterior condylar axis[7]. Locating the medial and lateral epicondyles precisely is often difficult to reproduce intraoperatively[8-10]. The measured resection technique has been proved that possibly lead to implant instability due to an increased incidence of femoral condylar lift-off when compared with the gap-balancing technique[6, 11]. Dennis analyzed 212 TKA patient's bony marker were registered before resection by computer navigation. The result showed a higher variability with femoral component position using the TEA and only $43 \%$ of cases matched the balanced alignment within \pm 3 , of which PC axis is $58 \%$ and AP axis is $39 \%[12]$.

Freeman et al. [13]first proposed the gap balancing technology in 1970 with the flexion gap first, based on which, Insall et al. [14]improved this technique and proposed the gap balance technology with Balancing Extension First. Gap balancing techniques rely on ligament releases prior to bone cuts. The limb could get correct approximate alignment before the determination of femoral component rotation by soft tissue releases. Some researches considered that gap balance can obtain more recent satisfaction from patients [15-17]. Appropriate soft tissue release and accurate osteotomy of the tibia are essential when surgeons selected to adopt gap balancing technique because the tibial resection will serve as a base and reference for the femoral bone resections,especially in the extension gap first technique. An inaccurate proximal tibial resection will lead to raised joint line\increased internal rotation or excessive external rotation of the femoral component or a mismatch of flexion and extension gap[18].

With the development of 3D printing technique and digital technique, patient-specific instrumentation (PSI) has been widely applied in orthopedic clinic[19], personalized printing resection blocks can be produced preoperatively based on MRI and CT data. But the accuracy of the PSI is still controversial. It has been proved that PSI combined digital technique can balance the precision,convenience and security to support complex and delicate surgery by some studies[20-22]. while other reports showed that PSI deviated from the surgical plan $10.5 \%$ in the coronal plane and $29.9 \%$ in the sagittal plane[23]. One of the causes for controversy is that most PSI systems used at present are only bone-referenced, and barely consider the functional parameters in combination[24, 25]. Appropriate soft tissue release is subjective and difficult to master in the surgery adopted gap balancing technique.

To solve the problems in gap balancing techniques, we used PSI combined with a new gap balancer device in TKA and compared it with the measured resection technique. There is three purpose of this study: The first one is to introduce a new, combination method for gap balancing in TKA. The second one 
is to introduce a new tool to perform the flexion gap balancing. The third one is to evaluate implant survivorship, patient outcomes, complications, and radiographic analysis in patients who underwent TKA of these two groups.

\section{Patients And Methods}

\section{Ethical approval}

Before we use the PSI and the new balancer device, we obtained approvals from the Changsha No. 3 hospital ethics committee and 3D printing technology medical application research institute of Changsha. A written informed consent was obtained from each patient in gap balancing group after the oral explanations on the detail of this study.

\section{Study design}

Data were obtained from the total knee arthroplasty finished in Changsha No. 3 hospital. 152 cases (150 knees) who underwent cemented primary TKA between August 2014 to June 2016. Most TKA finished during this period adopted two surgical techniques: the first one is traditional measured resection technique and the other one is gap balanced technique assisted by a new balancer device and PSI. We collected the clinical data of these patients for retrospective analysis. The study enrolled patients aged 22-85 years, inclusive with noninflammatory degenerative joint disease that was suitable candidates for cemented primary TKA. Exclusion criteria were as follows: Patients who had inflammatory arthritis, psychosocial disorders limiting rehabilitation, previous knee arthroplasty (including unicomartmental, biocompartmental or patellofemoral joint arthroplasty), prior patellectomy, prior high tibial osteotomy, or primary TKA in the affected knee and postoperative follow-up less than 3 years. Finally, 150 penitents (152 knees) were studied. Measured resection technique was adopted intraoperation in 70 patients (70 knees) and gap balanced technique assisted by a new balancer device and PSI was adopted intraoperation in 80 patients ( 82 knees). We collected the data of patient demographics (gender, age, $\mathrm{BMI}$ ), Knee Society score (KSS), alignment and deformity details pre-operation and the flexion of knee, VAS pain scores and radiographic photoporation at 12 weeks and 36 weeks.

The flexion of knee, Knee Society score (KSS), VAS pain scores and radiographic assessments were assessed preoperatively and postoperatively at 6 weeks, 12 weeks, 1 year, and then annually. The mechanical leg axis hip-knee angle (HKA) was measured on a lower extremity long-standing radiograph. Mechanical axis of the lower limb was measured using digital radiographs and special software (PACS, BOWEI Electronic Information, Hunan, China). All the measurements of radiograph were performed by an independent physician.(Table 1)

Table 1 Demographic data and preoperative status and alignment and deformity pre-operation 


\begin{tabular}{|lllll|}
\hline Variable & Total & GB group $(\mathrm{n}=82)$ & MR group $(\mathrm{n}=70)$ & p value \\
\hline Age & $67 \pm 11.3$ & $70.2(55$ to 76$)$ & $71.1(52$ to 78$)$ & 0.42 \\
\hline Gender (female) & $152(62.32 \%)$ & $62 \bigotimes 75.61 \% \bigotimes$ & $48 \varangle 68.57 \% \rrbracket$ & \\
\hline BMI & $24.30 \pm 3.99$ & $24.12 \pm 3.79$ & $24.37 \pm 4.01$ & 0.38 \\
\hline KSS knee score & $37.13 \pm 21.81$ & $39.03 \pm 21.92$ & $35.72 \pm 19.16$ & 0.31 \\
\hline Preoperative flexion & $91.9 \pm 17.4$ & $92.2^{\circ} \pm 15.4^{\circ}$ & $90.3 \pm 17.2^{\circ}$ & 0.57 \\
\hline Alignment & & & & \\
\hline Valgus:n(mean alignment) & $41 \rrbracket-10.8 \pm 6.5 \rrbracket$ & $17(-7.9 \pm 5.1)$ & $24(-12.7 \pm 6.3)$ & 0.34 \\
\hline Neutral:n & 20 & 8 & 12 & 0.56 \\
\hline Varus:n(mean alignment) & $91 \rrbracket 6.0 \pm 2.1 \rrbracket$ & $57(5.9 \pm 1.7)$ & $34(6.2 \pm 2.3)$ & 0.51 \\
\hline
\end{tabular}

\section{The new gap balancer device tool (gap balancer)}

The gap balance tool was designed by the orthopedics research team of Changsha No. 3 hospital. The balancer device is consisting of three parts: a handle with holes to place the line device connect with a lower platform plate, an inverse "U" like balancer pole with scale on both lateral and medial part to measure the gap and a teetertotter condyle holder. This device is ultimately designed to permit surgeons to find a line paralleled with tibial plate under a proper tension in $90^{\circ}$ flexion, the balancer device was sterilized by a plasma sterilizer in order to be used during the operation. Our group obtained invention patent of this balancer device (patent number冈201820329898.0).

\section{Preparation of PSI}

The patients were examined by CT scan before operation and then imaged with 64-row volumetric CT (SOMATOM Sensation 40, Siemens, Malvern, PA) with $5 \mathrm{~mm}$ slice thickness. The images were stored in DICOM format and analyzed by the mimics 17.0 (Materialise, Belgium). The angle and plate of both tibia and femoral distal bone resection and the prosthetic components size were designed before surgery by 3D printing technology medical application research institute of Changsha and printing by Beijing Engineering Technology R\&D Center. The objective was to achieve a neutral mechanical axis for the femur and tibia. Planning was reviewed and confirmed by the surgeon in each case. The resection plates of the tibia were designed at a $90^{\circ}$ angle to the longitudinal tibial axis with $3^{\circ}$ posterior slope. The flexion degree in the sagittal plane for the femoral component was depending on personal anatomical features. The templates were sterilized by a plasma sterilizer before surgery. Our group obtained invention patent of these PSI and the design method (patent number ZL201520623218.2 and ZL201510507788.X).

\section{Surgery}


All knees were operated through a standard midline incision and a medial parapatellar arthrotomy, using a cruciateretaining prosthesis(Smith \& Nephew Legion囚LINK and AKMEDICAL).

GB Group PSI were used to perform resection of the proximal tibia and femur distal. Anterior dislocated the tibia and to place the line device to make sure again that the osteotomy plane of the tibia is perpendicular to the anatomical axis of the tibia. It is critical to remove all osteophytes before releasing the soft tissue, including posterior femoral and tibial osteophytes. Then released the soft tissue to achieve a symmetrical extension gap. Distal femoral and proximal tibial cuts are measured by the balancer device (Figure 1) so that the cut surfaces are parallel. No soft tissue release after this step. Subsequently, the knee is brought into $90^{\circ}$ flexion, balancer device is introduced and tensed to make sure the medial and lateral balance is equal $ه$ then mark a line on the bone parallel to the pole in the balancer device. Appropriate 4-in-1 resection block post parallel to the line mark before, and the block is utilized to perform anterior, posterior, and chamfer bone cuts. Measuring and recording the lateral and medial gap by the calibrations on the balancer device.

MR Group After adequate exposure of the knee, an extramedullary guide was used to perform resection of the proximal tibia and femur distal. Femur is drilled in order to introduce internal femoral alignment rod into the intramedullary canal, followed by distal cutting block with preset parameters. Posterior referencing cutting block is utilized to identify the potential component size. And then an appropriate 4-in1 resection block is utilized to perform anterior, posterior, and chamfer bone cuts. Previously decided trial sizes and polyethylene are introduced and the knee is evaluated for tracking stability in the AP and Varus and valgus planes for balance.

The processing for tibial plateau, patella and patellar tracking of both GB and MR group is consistent with traditional surgeries.

\section{Statistical analysis}

Data were stored and analyzed with use of SPSS 24.0 software (SPSS INC., Chicago, IL, USA). Demographic data were presented as mean \pm standard deviation(SD). The categorical variables were compared with a chi-square or Fisher's exact tests. Differences and correlations of p $₫ 0.05$ were considered statistically significant.

\section{Results}

\section{Patient Outcomes}

Between August 2014 and June 2016 \a total of 150 patients underwent 152 TKAs at our center. Mean age was $67 \pm 11.3$ years (ranging from 52 to 78 years). In total, of patients (150) In total, $75.61 \%$ of patients (62 patients) were female in GB group and $68.57 \%$ of patients (48 patients) were female in MR group. 
The follow-up ranged from 35 to 52 months (mean 45 months). Until the latest follow-up, the mean ROM was not significantly different between the measured resection and gap-balancing group at 12 weeks $\left(100.2^{\circ} \pm 11.3^{\circ} \mathrm{vs} .99 .3^{\circ} \pm 13.2^{\circ}, \mathrm{p} \otimes 0.527\right)$ and 36 weeks $\left(109.4^{\circ} \pm 9.4^{\circ} \mathrm{vs} .110^{\circ} \pm 12.1^{\circ}, \mathrm{p} \otimes 0.490\right)$. There were no significant differences between the measured resection and gap-balancing groups in terms of the KSS scores $(82.75 \pm 20.98$ vs. $81.29 \pm 19.67$ points, $p \otimes 0.712)$ or the VAS pain scores at 12 weeks $(1.57 \pm 2.91$ vs. $2.67 \pm 2.29$ points, $\mathrm{p} \otimes 0.496)$ and the KSS scores ( $92.19 \pm 19.11$ vs. $88.17 \pm 22.45$ points, $\mathrm{p} \llbracket 0.623$ ) or the VAS pain scores at 36 weeks $(1.37 \pm 2.23$ vs. $2.10 \pm 2.45$ points, $p \llbracket 0.414)$.

\section{Prosthesis Survivorship}

As of this writing, the prosthesis survivorship was $99.5 \%$ in GB group and $100 \%$ in MR group. One patient underwent revision surgery in gap balance group at 2 years postoperatively due to infection. At the final follow-up, the patients got KSS scores at 82 points and had no further sequela.

\section{The accuracy of PSI and balancer device}

The operation time of the GB group averaged 51 minutes $₫ 41-69$ minutes $₫$ and 58 minutes $₫ 43-67$ minutes $\rrbracket$ of the MR group $₫$ without significant difference between two groups $(P \otimes 0.05)$. And the time required to balance the flexion gap averaged 2 minutes. We consider the measuring procedure as easy to perform with the help from the gap balancer device we designed. In GB group, all cases received the TKA and implanted prosthesis with PSI, and the balancer device was used for gap balancing as planned. In 3 cases, the tibial component used intro-operation is one size smaller than planned.

\section{Complications}

The complications developed after operation in 4 cases in GB group $₫ 4 / 82 \rrbracket a n d 6$ in MR group $\varangle 6 / 70 \rrbracket$,

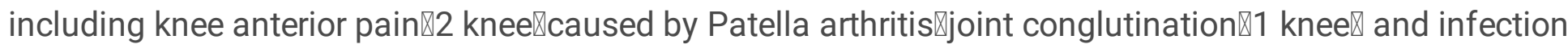
$\bigotimes 1$ knee『in GB group and knee anterior pain $₫ 5$ knee『caused by abnormal patellar tracking $邓 j o i n t$ conglutination $₫ 1$ knee囚in MR group. There were no intraoperative complications.

\section{Radiographic Analysis}

The radiographic evaluation at latest follow-up did not demonstrate any evidence of progressive radiolucencies, loosening, or subsidence of any prosthesis except for the case of infection. The average joint line displacement in GB group was $1.3 \pm 1.1 \mathrm{~mm}$ (range $0-3$ ) proximal and $1.2 \pm 1.4 \mathrm{~mm}$ in MR group. No outliers $₫ 5 \mathrm{~mm}$ in each group were record.

The mean leg axis was $1.8^{\circ} \pm 1.5^{\circ}$ varus (range $0^{\circ}-3^{\circ}$ varus) versus the neutral mechanical axis in GB group and $1.4^{\circ} \pm 1.2^{\circ}$ 冈range $0^{\circ}-3^{\circ} \otimes$ in MR group. No outliers with $\otimes 3^{\circ}$ deviation in each group were record.

\section{Table 2 Clinical and radiographic outcome data at 12 weeks and 36 weeks}




\begin{tabular}{|llll|}
\hline Variable & GB group $(\mathrm{n}=82)$ & MR group $(\mathrm{n}=70)$ & p value \\
\hline Joint line displacement $(\mathrm{mm})$ & $1.3 \pm 1.1$ & $1.2 \pm 1.4$ & 0.391 \\
\hline Flexion at 12 weeks & $100.2^{\circ} \pm 11.3^{\circ}$ & $99.3^{\circ} \pm 13.2^{\circ}$ & 0.527 \\
\hline Flexion at 36 weeks & $109.4^{\circ} \pm 9.4^{\circ}$ & $110^{\circ} \pm 12.1^{\circ}$ & 0.490 \\
\hline hip-knee angle $\left(^{\circ}\right)$ & $1.8^{\circ} \pm 1.5^{\circ}$ & $1.4^{\circ} \pm 1.2^{\circ}$ & 0.556 \\
\hline KSS knee score at 12 weeks & $82.75 \pm 20.98$ & $81.29 \pm 19.67$ & 0.712 \\
\hline KSS knee score at 36 weeks & $92.19 \pm 19.11$ & $88.17 \pm 22.45$ & 0.623 \\
\hline VAS at 12 weeks & $1.57 \pm 2.91$ & $2.67 \pm 2.29$ & 0.496 \\
\hline VAS at 36 weeks & $1.37 \pm 2.23$ & $2.10 \pm 2.45$ & 0.414 \\
\hline
\end{tabular}

\section{Discussion}

Currently, standard TKA techniques utilize either measured resection or gap-balancing methods to determine femoral component rotation. Bone cuts are made in preference to soft tissue tension in measured resection technique prefer. While『gap balancing techniques rely on ligament releases prior to bone cuts. But the best method for obtaining the rotational alignment of the femoral component during flexion remains controversial. Gap Balancing technique is supported by many surgeons that can get a symmetric, rectangular flexion space intraoperation. Although gap balancing technique is effective, there still exist some difficult problems in dispute, which need further discussion.

First, Gap balancing technique has been thought to sacrifices joint-line maintenance in order to improve gap symmetry. The elevation of joint-line may due to significantly greater distal femoral resection and larger tibial insert thickness[27]. Different from the measured resection technique performed femur and tibial resection independently, the osteotomy of the femoral anteroposterior condyle in the gap balance technique is performed reference to the proximal tibia resection. Improper tibial resection would also lead to elevation of joint-line or mismatch of flexion and extension gap dimensions. So, an accurate proximal tibial and femur distal cut is crucial.

In this study, Data of 152 patients received TKA from August 2014 to June 2016 were analyzed retrospectively, and we provide an intraoperative comparison of the GB (assisted by PSI and new balancer device) and MR techniques for establishing femoral component position. The resection of the proximal tibia and femur distal were performed by PSI which were designed according to individual anatomical characteristic and has already been proved to be accuracy and safety in part of orthopedic surgery[2832]. We research team have a lot of experience on digital orthopedics [33]. In this study, alignment of the tibial component with no deviations $\otimes 1.5^{\circ}$ compared to planning. there was no relevant displacement of the joint line $₫ 3 \mathrm{~mm}$ in both two group, there are 15 cases at $3 \mathrm{~mm}$ in GB group and 12 cases in MR group 


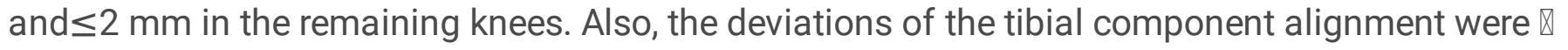
$1.5^{\circ}$ compared to planning with the assistants of PSI.

Another critical procedure in gap balance technique is the femoral component rotation. Many principles and surgical devices for ligament balance during TKA have been developed. Different spacers, including trial components and blocks may assist in stretching the ligaments. The medial and lateral lift-off can then be measured by surgeons' eyes and experience or indirectly by a navigator. Tensors and spreaders apply tension to the ligaments in a controlled manner with or without electric instruments measures compressive loads. Most of these devices are expensive, add to the complexity of the surgery and are time consuming.

The new balancer device we designed and used in surgery is like a seesaw, it can find a bony landmark paraclete to the posterior condylar reresection plate and confirm the femoral component rotation. We consider the biggest advantage of the balancer device is easy to perform the flexion balance procedure, and the time to confirm the femoral component rotation take no more than 2-3 min. The scale on the condylar holder clearly shows the lateral and medial gap height. Clinical and radiographic outcome data at 12 weeks and 36 weeks demonstrated that patients can get satisfied function from the gap balancing technique assisted by the PSI and new balancer device. There are some disadvantages that need to be addressed and improved: we cannot know joint distraction force explicitly; it will be more precise if there is a grip dynamometer connect to the balancer device.

We achieved the goal of avoiding anatomical differences, finding an easy way to get a symmetric, rectangular flexion space intraoperation and restoration the natural joint line by using the PSI and the balancer device in this study. Our analysis showed that both the measured resection and combination techniques can be used to achieve accurate femoral component alignment and similar 3 years outcomes. There is no different from previous studies[34, 35].

\section{Conclusion}

In conclusion, the gap balancing technique assisted by the new balancer device and PSI can be used to achieve accurate femoral component alignment as well as measured resection in 3 years outcomes. The new balancer device can be takes into account by surgeons who prefer the gap-balancing technique together with the PSI.

There is some limitations of this study: first, it is a retrospective study, we cannot compare the femoral component rotation angle with the bony marker. Second, the follow-up period is the short relatively. Consequently, more scientific and valuable research need to perform.

\section{Declarations}

\section{Acknowledgements}


None.

\section{Funding}

Science and Technology Innovation Plan Project of Hunan Province, China [2017JJ2284]

Science and Technology Plan Project of Hunan Province, China [2018SK4014]

\section{Availability of data and materials}

Not applicable.

\section{Authors' contributions}

Ting Deng and Tangyou Liu contributed equally to this study. Qing Lei is corresponding author of the paper. All authors read and approved the final manuscript.

\section{Authors' information}

Ting Deng, Tangyou Liu, Qing Lei \& Song Chen are members of Department of Orthopaedics, The Third Hospital of Changsha, Changsha, China. Lihong Cai is a member of Department of Radiology, The Third Hospital Of Changsha, Changsha, China

\section{Competing interests}

The authors declare that they have no competing interests.

\section{Consent for publication}

Not applicable.

\section{Ethics approval and consent to participate}

Not applicable.

\section{References}

1. Hube R, et al. [Extension first technique for TKA implantation]. Oper Orthop Traumatol. 2011;23(3):241-8.

2. Czurda $T$, et al. The association between component malalignment and post-operative pain following navigation-assisted total knee arthroplasty: results of a cohort/nested case-control study. Knee Surg Sports Traumatol Arthrosc. 2010;18(7):863-9.

3. Ritter MA, et al. The effect of alignment and BMI on failure of total knee replacement. J Bone Joint Surg Am. 2011;93(17):1588-96. 
4. Heesterbeek PJ, Jacobs WC, Wymenga AB. Effects of the balanced gap technique on femoral component rotation in TKA. Clin Orthop Relat Res. 2009;467(4):1015-22.

5. Hube R, et al. [Extension first technique for TKA implantation]. Oper Orthop Traumatol. 2011;23(3):241-8.

6. Daines BK, Dennis DA. Gap balancing vs. measured resection technique in total knee arthroplasty. Clin Orthop Surg. 2014;6(1):1-8.

7. Franceschini V, Nodzo SR, Gonzalez DVA. Femoral Component Rotation in Total Knee Arthroplasty: A Comparison Between Transepicondylar Axis and Posterior Condylar Line Referencing. J Arthroplasty. 2016;31(12):2917-21.

8. Hatayama K, et al. Relationship between femoral component rotation and total knee flexion gap balance on modified axial radiographs. J Arthroplasty. 2011;26(4):649-53.

9. Nagamine R, et al. Reliability of the anteroposterior axis and the posterior condylar axis for determining rotational alignment of the femoral component in total knee arthroplasty. $\mathrm{J}$ Orthop Sci. 1998;3(4):194-8.

10. Pagnano MW, Hanssen AD. Varus tibial joint line obliquity: a potential cause of femoral component malrotation. Clin Orthop Relat Res, 2001(392): p. 68-74.

11. Dennis DA, et al. Gap balancing versus measured resection technique for total knee arthroplasty. Clin Orthop Relat Res. 2010;468(1):102-7.

12. Dennis DA. Measured resection: an outdated technique in total knee arthroplasty. Orthopedics. 2008;31(9):940, 943-4.

13. Freeman MA, et al., Knee arthroplasty at the London Hospital. 1975-1984. Clin Orthop Relat Res, 1986(205): p. 12-20.

14. Insall J, et al., Total condylar knee replacment: preliminary report. Clin Orthop Relat Res, 1976(120): p. 149-54.

15. Dennis DA. Measured resection: an outdated technique in total knee arthroplasty. Orthopedics. 2008;31(9):940, 943-4.

16. Griffin FM, Insall JN, Scuderi GR. Accuracy of soft tissue balancing in total knee arthroplasty. J Arthroplasty. 2000;15(8):970-3.

17. Yau WP, Chiu KY, Tang WM. How precise is the determination of rotational alignment of the femoral prosthesis in total knee arthroplasty: an in vivo study. J Arthroplasty. 2007;22(7):1042-8.

18. Fu Y, et al. Alignment outcomes in navigated total knee arthroplasty: a meta-analysis. Knee Surg Sports Traumatol Arthrosc. 2012;20(6):1075-82.

19. Daniilidis K, Tibesku CO. Frontal plane alignment after total knee arthroplasty using patient-specific instruments. Int Orthop. 2013;37(1):45-50.

20. Laende EK, Richardson CG, Dunbar MJ. A randomized controlled trial of tibial component migration with kinematic alignment using patient-specific instrumentation versus mechanical alignment using computer-assisted surgery in total knee arthroplasty. Bone Joint J, 2019. 101-B(8): p. 929-940. 
21. Yamamura K, et al., Design improvement in patient-specific instrumentation for total knee arthroplasty improved the accuracy of the tibial prosthetic alignment in the coronal and axial planes. Knee Surg Sports Traumatol Arthrosc, 2019.

22. Attard A, et al. Health costs and efficiencies of patient-specific and single-use instrumentation in total knee arthroplasty: a randomised controlled trial. BMJ Open Qual. 2019;8(2):e000493.

23. Thienpont E, Schwab PE, Fennema P. Efficacy of Patient-Specific Instruments in Total Knee Arthroplasty: A Systematic Review and Meta-Analysis. J Bone Joint Surg Am. 2017;99(6):521-30.

24. Mont MA, et al. Patient specific instrumentation. J Arthroplasty. 2014;29(9):1693.

25. Maus $U$, et al. No improvement in reducing outliers in coronal axis alignment with patient-specific instrumentation. Knee Surg Sports Traumatol Arthrosc. 2018;26(9):2788-96.

26. Cooke TD, Li J, Scudamore RA. Radiographic assessment of bony contributions to knee deformity. Orthop Clin North Am. 1994;25(3):387-93.

27. Babazadeh S, et al. Gap Balancing Sacrifices Joint-Line Maintenance to Improve Gap Symmetry: 5Year Follow-Up of a Randomized Controlled Trial. J Arthroplasty. 2018;33(1):75-8.

28. Daniilidis K, Tibesku CO. Frontal plane alignment after total knee arthroplasty using patient-specific instruments. Int Orthop. 2013;37(1):45-50.

29. Heyse TJ, Tibesku CO. Improved tibial component rotation in TKA using patient-specific instrumentation. Arch Orthop Trauma Surg. 2015;135(5):697-701.

30. Heyse TJ, Tibesku CO. Improved femoral component rotation in TKA using patient-specific instrumentation. Knee. 2014;21(1):268-71.

31. Daniilidis K, Tibesku CO. A comparison of conventional and patient-specific instruments in total knee arthroplasty. Int Orthop. 2014;38(3):503-8.

32. Boonen B, Schotanus MG, Kort NP. Preliminary experience with the patient-specific templating total knee arthroplasty. Acta Orthop. 2012;83(4):387-93.

33. Deng $T$, et al. The accuracy and the safety of individualized 3D printing screws insertion templates for cervical screw insertion. Comput Assist Surg (Abingdon). 2016;21(1):143-9.

34. Hommel H, Perka C. Gap-balancing technique combined with patient-specific instrumentation in TKA. Arch Orthop Trauma Surg. 2015;135(11):1603-8.

35. Churchill JL, et al. Gap-Balancing versus Measured Resection Technique in Total Knee Arthroplasty: A Comparison Study. J Knee Surg. 2018;31(1):13-6.

\section{Figures}




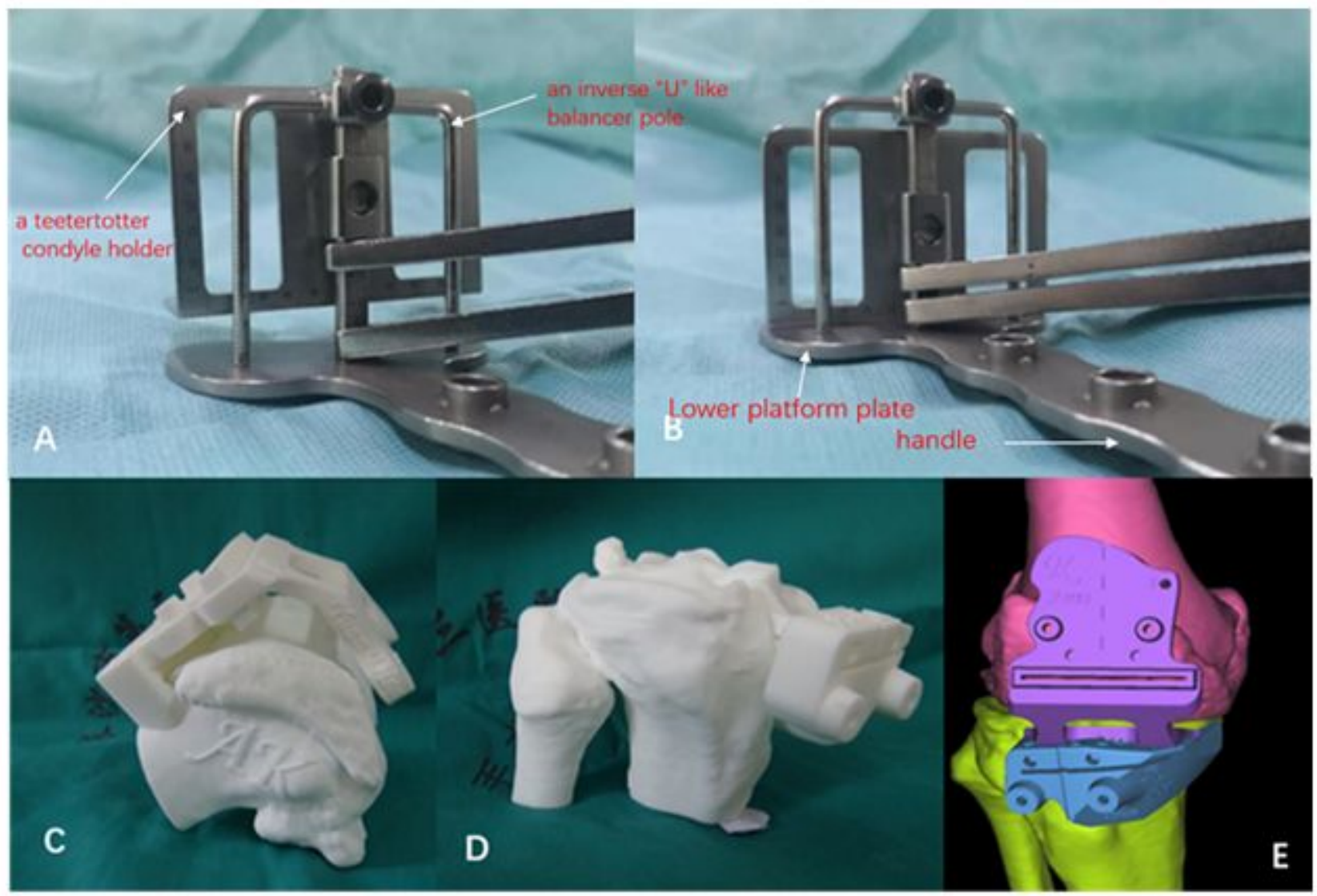

\section{Figure 1}

The new gap balance tool (A, B). PSI (C is tibia component; $D$ is femur component). The planned resection trajectories of femur and tibia(E)

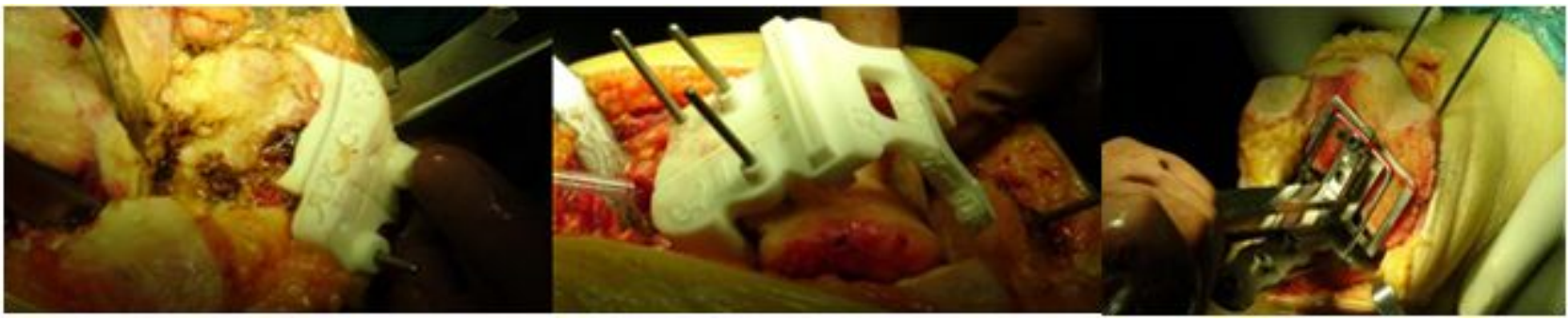

\section{Figure 1}

Intraoperative photograph of the surgery procedure of gap balancing group ( $A$ is resection of the proximal tibia assisted by PSI; $B$ is resection of the femur distal assisted by PSI C is the flexion gap balancing assisted by the new balancer device we designed). 